\title{
Erratum
}

\section{A unified approach to concrete Plancherel theory of homogeneous spaces}

\author{
manuscripta math. 94, 133-149 (1997)
}

\section{Ronald L. Lipsman}

Department of Mathematics, University of Maryland, College Park, MD 20742, USA.

e-mail: rll@math.umd.edu

28 January 1998

\section{Note by the editors}

We regret that, due to certain unforeseen font problems, there have occurred a number of misprints in the above-mentioned paper, which was published in October 1997. Those misprints may render part of that paper unintelligble, and therefore we publish here the list of necessary corrections, as provided by the author.

\section{List of corrections}

In the following, page numbers and line numbers etc. refer to the abovementioned article.

Page 135, line 12 from below: Replace $\mathcal{D}(\mathcal{G})$ by $\mathcal{D}(G)$ (two times). Thus the letter $\mathcal{G}$ has to be replaced by $G$. The same misprint has occurred also in formulas (2.1), (2.2), (2.3), (5.3), and on p. 145 in the last displayed formula (which is not numbered).

Page 135, line 8 from below: In this displayed formula, the symbol $\backslash_{\pi}$ has to be replaced by $n_{\pi}$, and the symbol $\lceil$ has to be replaced by $d$. Thus the correct formula reads:

$$
\mathcal{H}_{\tau}^{\infty}=\int^{\oplus} n_{\pi} \mathcal{H}_{\pi}^{\infty} d \mu(\pi) \quad \mathcal{H}_{\tau}^{-\infty}=\int^{\oplus} n_{\pi} \mathcal{H}_{\pi}^{-\infty} d \mu(\pi) .
$$

The misprint $\lceil$ for $d$ has also occurred on p. 138 (last displayed formula). 
Page 135, line 7 from below: In the formula which extends to the next line, the exponent $(\cdots)^{\mathcal{H}, \amalg^{-\infty / \epsilon}}$ is to be replaced by $(\cdots)^{H, q^{-1 / 2}}$; moreover, the symbol $\langle$ is to be replaced by $h$, and at formula's end $\mathcal{H}$ by $H$. Thus the correct formula reads:

$$
\left(\mathcal{H}_{\tau}^{-\infty}\right)^{H, q^{-1 / 2}}=\left\{\alpha \in \mathcal{H}_{\pi}^{-\infty}: \pi(h) \alpha=q^{-1 / 2}(h) \alpha, \forall h \in H\right\}
$$

The above mentioned misprint in the exponent has also occurred on p. 136 (in Theorem 2.1); p. 137 (four times in displayed formulas); p. 144 (second displayed formula); p. 145 (line 4).

Page 141, line 4: The formula on this line has to be replaced by

$$
f: G \rightarrow \mathbb{C}, f(v g)=\lambda(v) f(g), v \in V, g \in G
$$

Page 143, Remarks 4.2: In these remarks, $\mathcal{D}(\mathcal{R})$ has to be replaced by $\mathcal{D}(R)$ (three times). In Remark 1, line 2, the formula extending to the next line is to be replaced by

$$
\mathcal{D}(R) \subset L^{2}(R, d r)^{\infty} .
$$

Page 145, line 3: In the formula extending to the next line, $\mathcal{V}$ has to be replaced by $V$; moreover, the wrong exponent has to be corrected as explained earlier. Hence the correct formula reads as follows:

$$
U_{\lambda}: \mathcal{H}_{\lambda}^{\infty} \rightarrow\left(\mathcal{H}_{\lambda}^{-\infty}\right)^{H, q^{-1 / 2}}, \lambda \in V .
$$

